\title{
Study Site Target Accrual Number Range
}

National Cancer Institute

\section{Source}

National Cancer Institute. Study Site Target Accrual Number Range. NCI Thesaurus.

Code $C 94153$.

A range of integers specifying the minimum and maximum number of subjects needed for enrollment at this site. 